Supplemental Information for:

\title{
Developing Structural First Principles for Alkylated Triphenylphosphonium-based Ionic Liquids
}

Brianna O'Rourke, ${ }^{\ddagger, a}$ Clare Lauderback, ${ }^{\ddagger}$ Lara I. Teodoro, ${ }^{\ddagger}$ a Morgan Grimm, ${ }^{a}$ Matthias Zeller, ${ }^{\mathrm{b}}$ Arsalan Mirjafari, ${ }^{*, c}$ Gary L. Guillet, ${ }^{\mathrm{d}}$ Patrick C. Hillesheim*a,c

${ }^{a}$ Department of Chemistry and Physics, Ave Maria University, Ave Maria, Florida, 34142, United States

${ }^{\mathrm{b}}$ Department of Chemistry, Purdue University, West Lafayette, Indiana 47907, United States

${ }^{\mathrm{c}}$ Department of Chemistry and Physics, Florida Gulf Coast University, Fort Myers, Florida, 33965, United States

${ }^{\mathrm{d}}$ Department of Chemistry and Biochemistry, Georgia Southern University, Savannah, Georgia, 31419, United States 
Table S1: Percent interactions of the cations for compounds $1-4$ as calculated from the Hirshfeld surfaces.

\begin{tabular}{|c|c|c|c|c|c|c|}
\hline & H...All & $\mathrm{H} \ldots \mathrm{H}$ & $\mathrm{H} . . . \mathrm{N}$ & $\mathrm{H} . . . \mathrm{O}$ & $\mathrm{H} \ldots \mathrm{F}$ & $\mathrm{H} . . \mathrm{C} \mid \mathrm{C} . . \mathrm{H}$ \\
\hline \multirow{3}{*}{$\begin{array}{l}\text { BETI } \\
\text { BuTPP Cation }\end{array}$} & 85.0 & 33.5 & 1.0 & 17.7 & 28.5 & 10.5 \\
\hline & C...All & C...O & C...F & & & \\
\hline & 15.0 & 0.9 & 7.9 & & & \\
\hline \multirow{4}{*}{$\begin{array}{l}\text { NCyF } \\
\text { BuTPP Cation } \\
1 \text { Part } 1 \\
\end{array}$} & H...All & $\mathrm{H} \ldots \mathrm{H}$ & $\mathrm{H} \ldots \mathrm{N}$ & $\mathrm{H} . . . \mathrm{O}$ & $\mathrm{H} \ldots \mathrm{F}$ & $\mathrm{H} \ldots \mathrm{C} \mid \mathrm{C} . . \mathrm{H}$ \\
\hline & 85.4 & 39.1 & 2.0 & 22.0 & 15.4 & 17.0 \\
\hline & C...All & C...O & C...F & C...N & C...C & \\
\hline & 14.6 & 1.0 & 2.1 & 0.4 & 1.1 & \\
\hline \multirow{4}{*}{$\begin{array}{l}\text { NCyF } \\
\text { BuTPP Cation } \\
2 \text { Part } 1 \\
\end{array}$} & H...All & $\mathrm{H} . . . \mathrm{H}$ & $\mathrm{H} . . . \mathrm{N}$ & $\mathrm{H} . . . \mathrm{O}$ & $\mathrm{H} . . . \mathrm{F}$ & H...C|C..H \\
\hline & 84.9 & 36.7 & 2.9 & 13.6 & 23.3 & 17.9 \\
\hline & C...All & C...O & C...F & C...N & C...C & \\
\hline & 15.1 & 2.1 & 1.7 & - & 1.6 & \\
\hline \multirow{4}{*}{$\begin{array}{l}\text { TF2N BuTPP } \\
\text { Cation } \\
\end{array}$} & H...All & $\mathrm{H} . . . \mathrm{H}$ & $\mathrm{H} . . . \mathrm{N}$ & $\mathrm{H} . . . \mathrm{O}$ & $\mathrm{H} . . . \mathrm{F}$ & H...C|C..H \\
\hline & 85.2 & 29.4 & 2.1 & 16.8 & 26.7 & 22.6 \\
\hline & C...All & C...O & C...F & C...N & C...C & \\
\hline & 14.8 & 0.9 & 1.5 & - & - & \\
\hline \multirow{4}{*}{$\begin{array}{l}\text { FSI BuTPP } \\
\text { Cation A }\end{array}$} & H...All & $\mathrm{H} . . . \mathrm{H}$ & $\mathrm{H} . . . \mathrm{N}$ & $\mathrm{H} . . . \mathrm{O}$ & $\mathrm{H} . . . \mathrm{F}$ & $\mathrm{H} \ldots \mathrm{C} \mid \mathrm{C} \ldots \mathrm{H}$ \\
\hline & 84.4 & 42.1 & 2.4 & 18.5 & 10.6 & 22.9 \\
\hline & C...All & C...O & C...F & C...N & C...C & \\
\hline & 15.6 & 0.9 & 2.4 & - & - & \\
\hline \multirow{4}{*}{$\begin{array}{l}\text { FSI BuTPP } \\
\text { Cation B }\end{array}$} & H...All & $\mathrm{H} . . . \mathrm{H}$ & $\mathrm{H} . . . \mathrm{N}$ & $\mathrm{H} . . . \mathrm{O}$ & $\mathrm{H} \ldots \mathrm{F}$ & $\mathrm{H} \ldots \mathrm{C} \mid \mathrm{C} \ldots \mathrm{H}$ \\
\hline & 85.2 & 46.4 & 2.4 & 20.8 & 6.6 & 21.4 \\
\hline & C...All & C...O & $C \ldots F$ & C...N & C...C & \\
\hline & 14.8 & 0.8 & 0.7 & 0.8 & - & \\
\hline
\end{tabular}


Table S2: Tabulated thermal data from all 3 cycles of DSC for the melting points Tm

\begin{tabular}{|c|c|c|c|c|}
\hline Compound & Cycle 1 & Cycle 2 & Cycle 3 & Average \\
\hline $\mathbf{1}$ & 90.05 & 89.18 & 89.20 & $89.47 \pm 0.41$ \\
\hline $\mathbf{2}$ & 85.20 & - & - & 85.20 \\
\hline $\mathbf{3}$ & 118.50 & - & - & 118.50 \\
\hline $\mathbf{4}$ & 126.44 & 126.02 & 126.05 & $126.17 \pm 0.19$ \\
\hline
\end{tabular}


NMR Spectra

Figure S1. 1H NMR for Compound 1

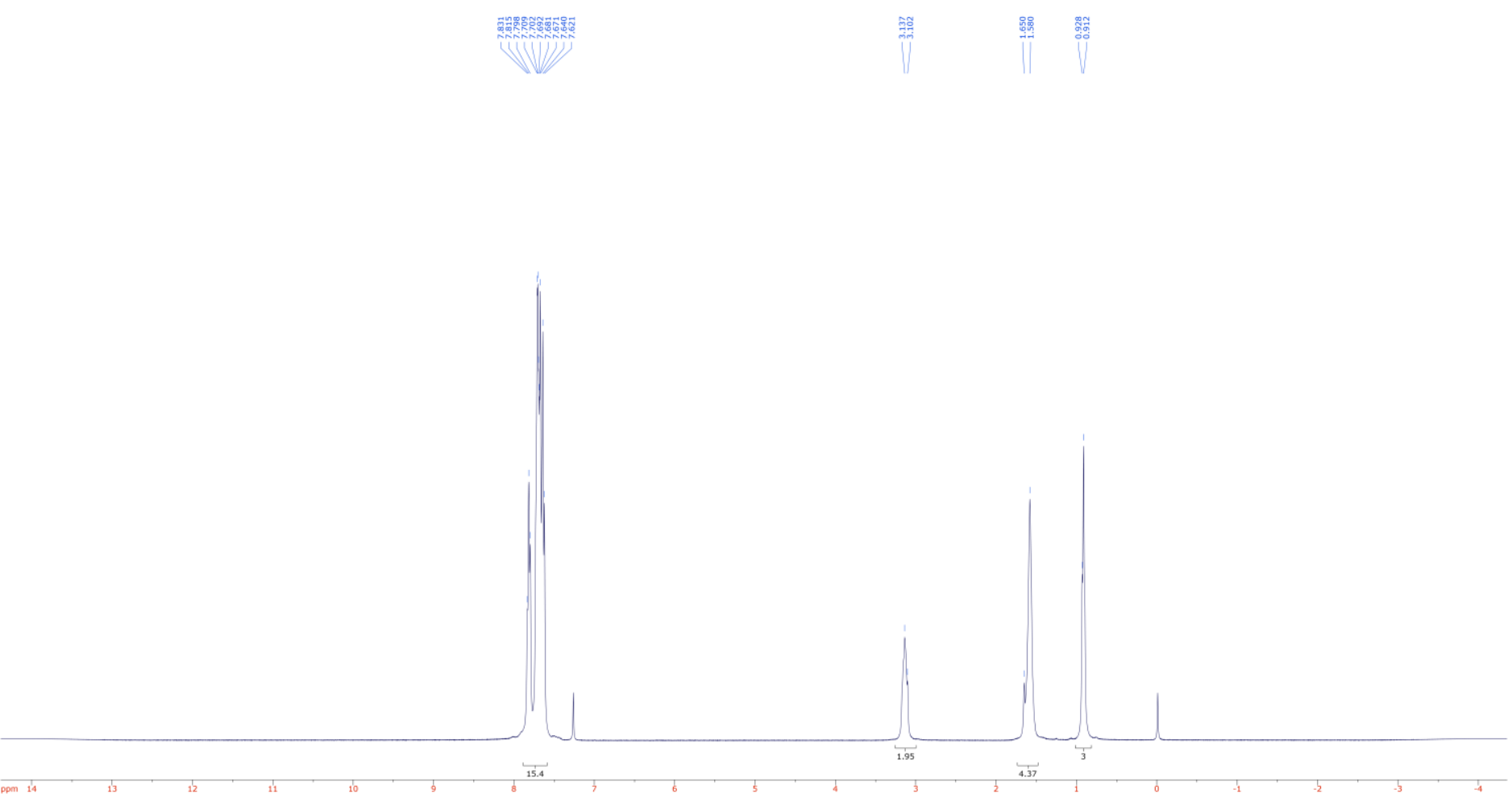

Figure S2. 13C NMR for Compound 1
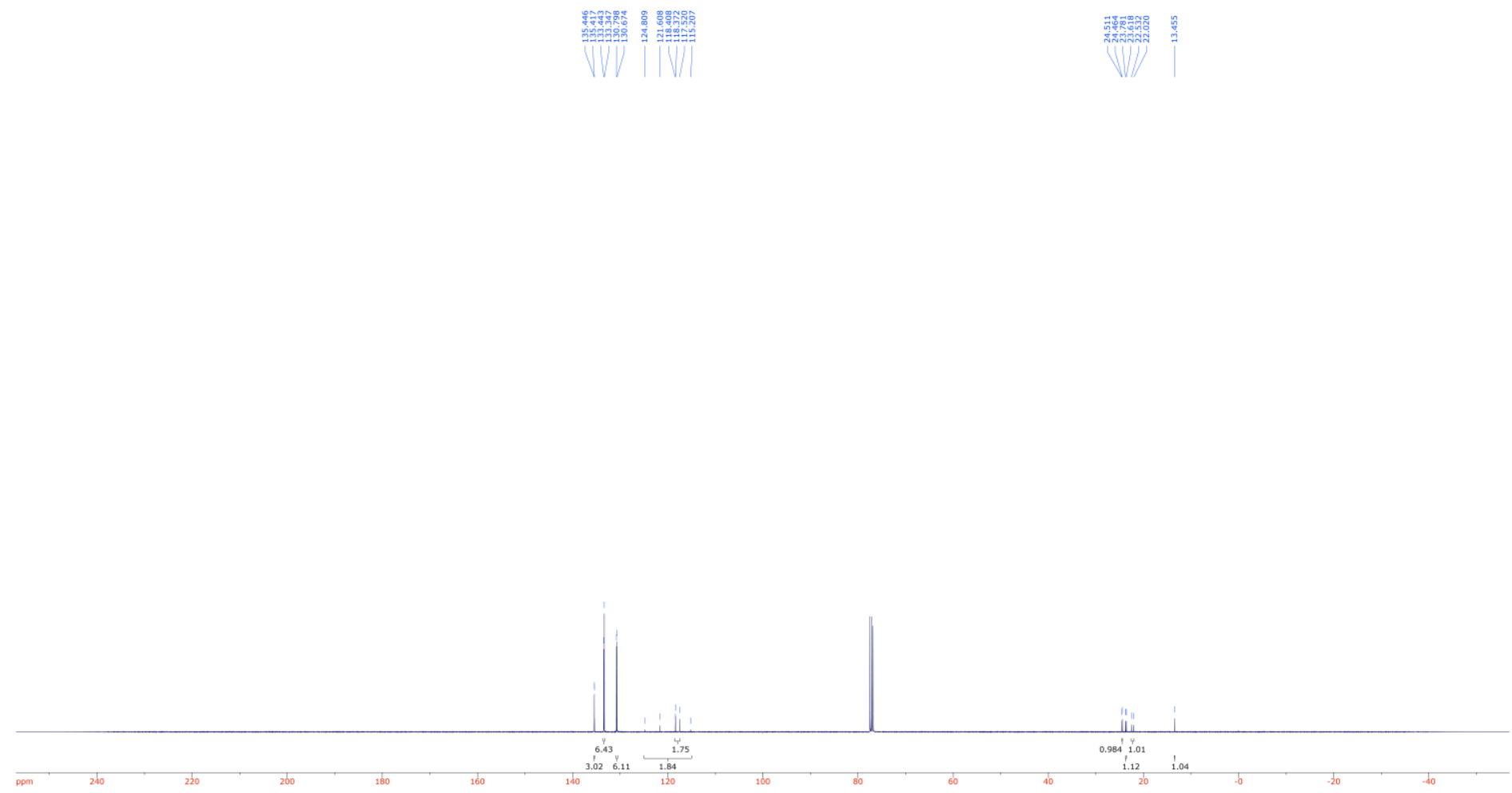
Figure S3: 19F NMR for Compound 1

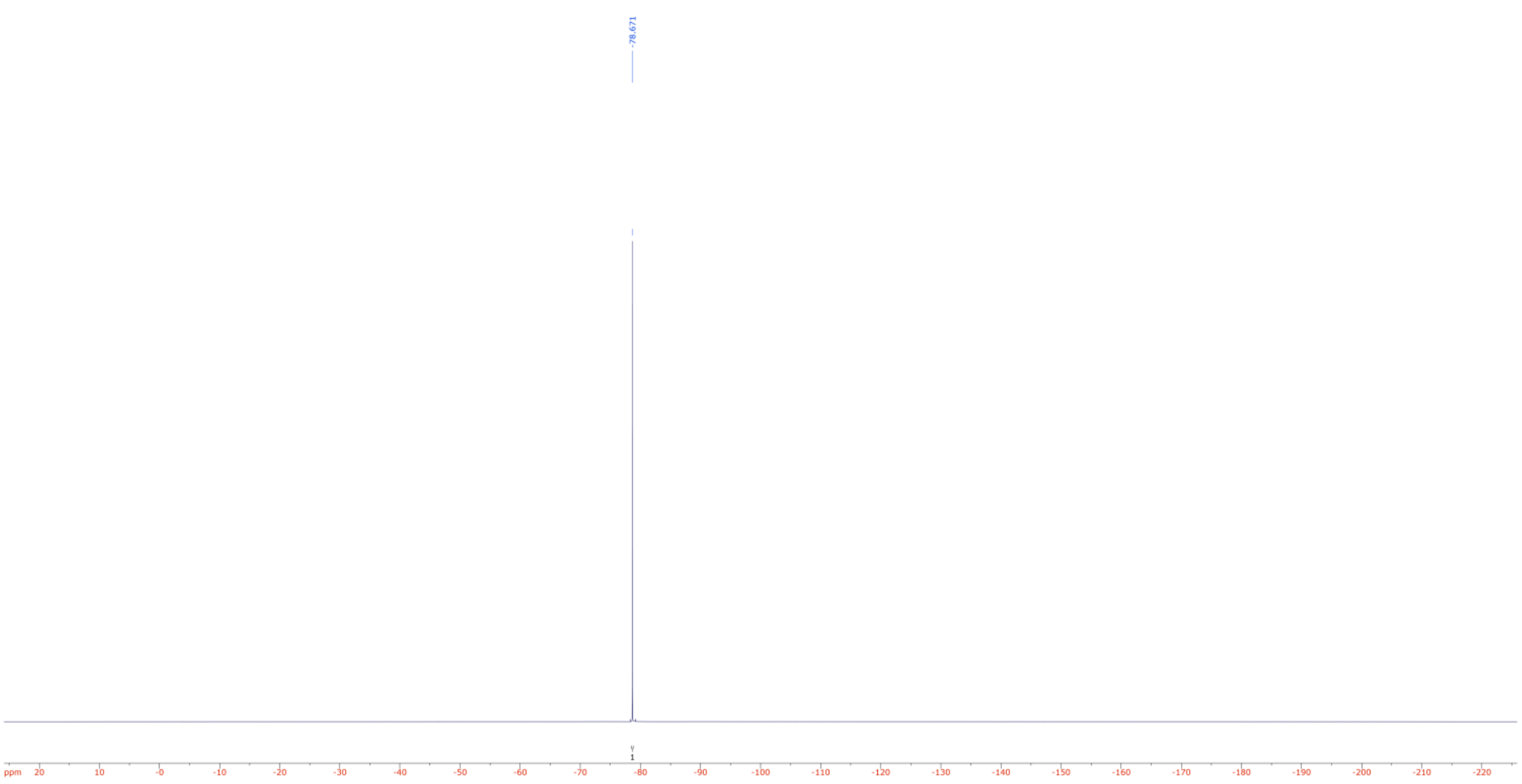

Figure S4. 1H NMR for Compound 2
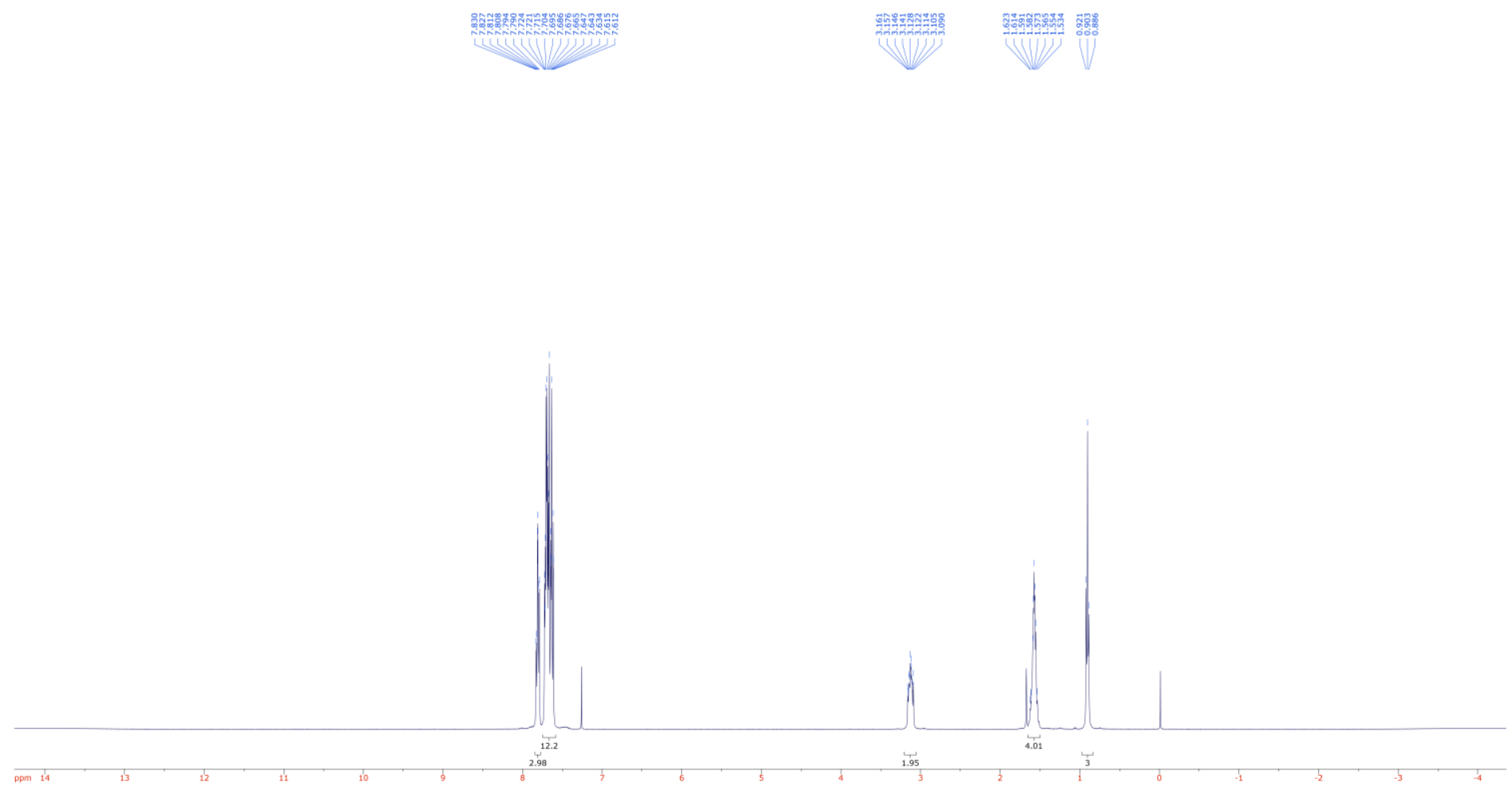
Figure S5: 13C NMR for Compound 2
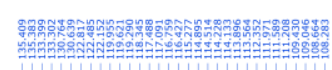

VU

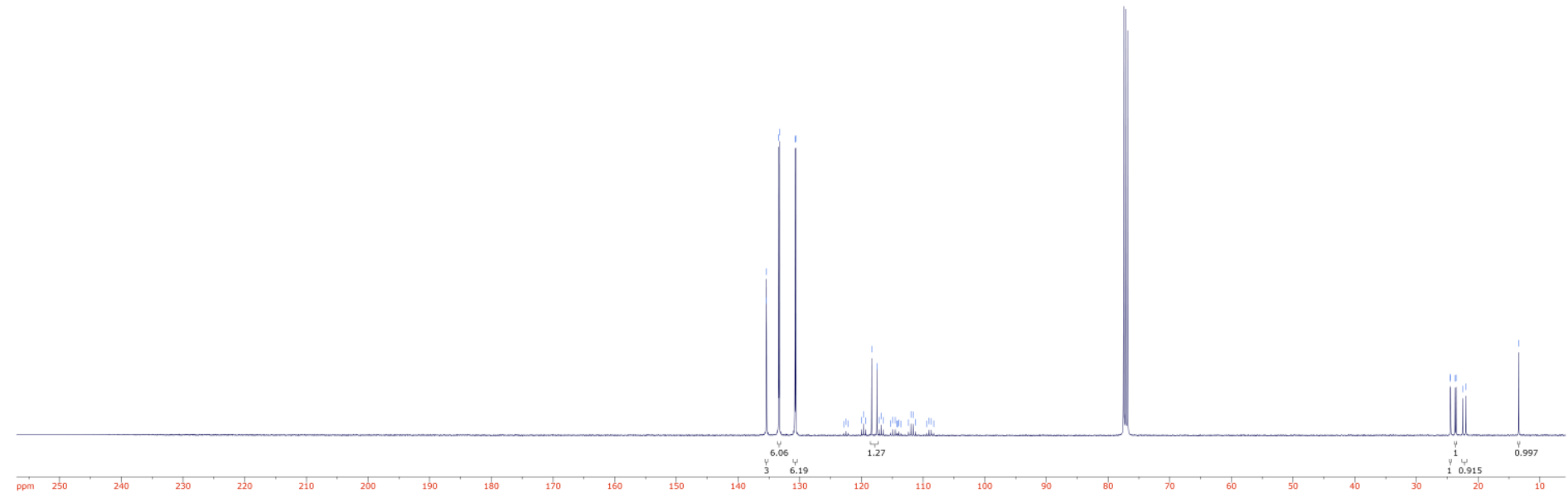

Figure S6: 19F NMR for Compound 2

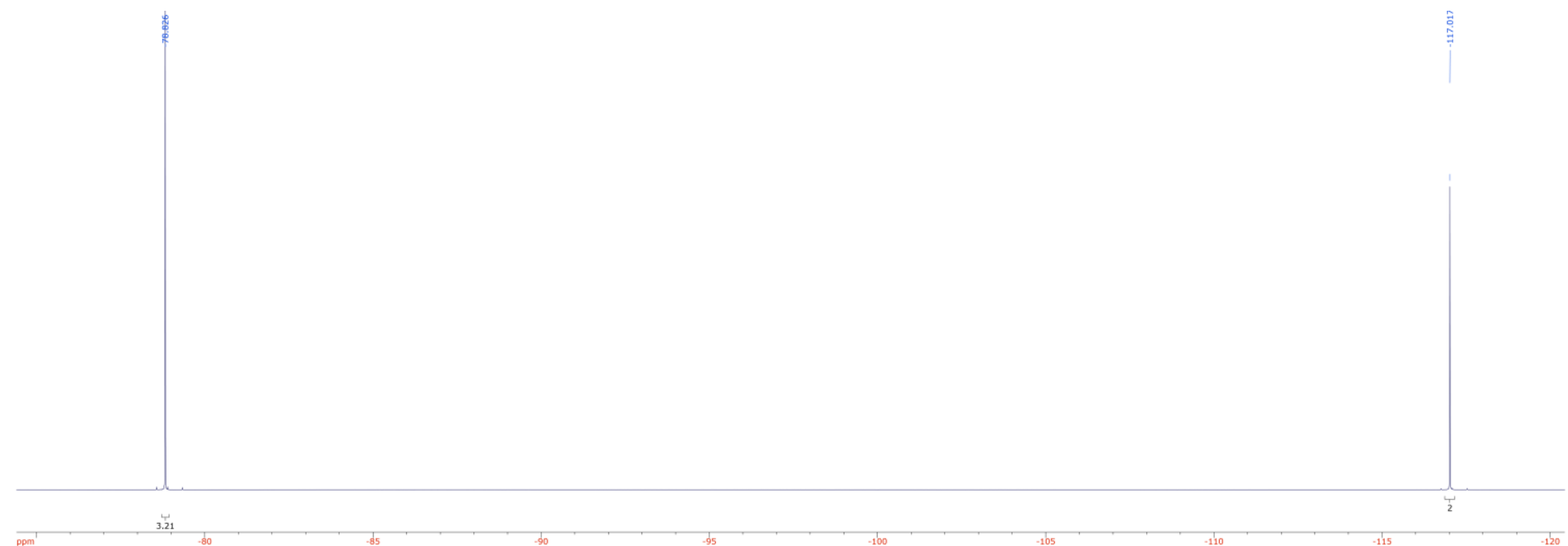


Figure S7: $1 \mathrm{H}$ NMR for Compound 3

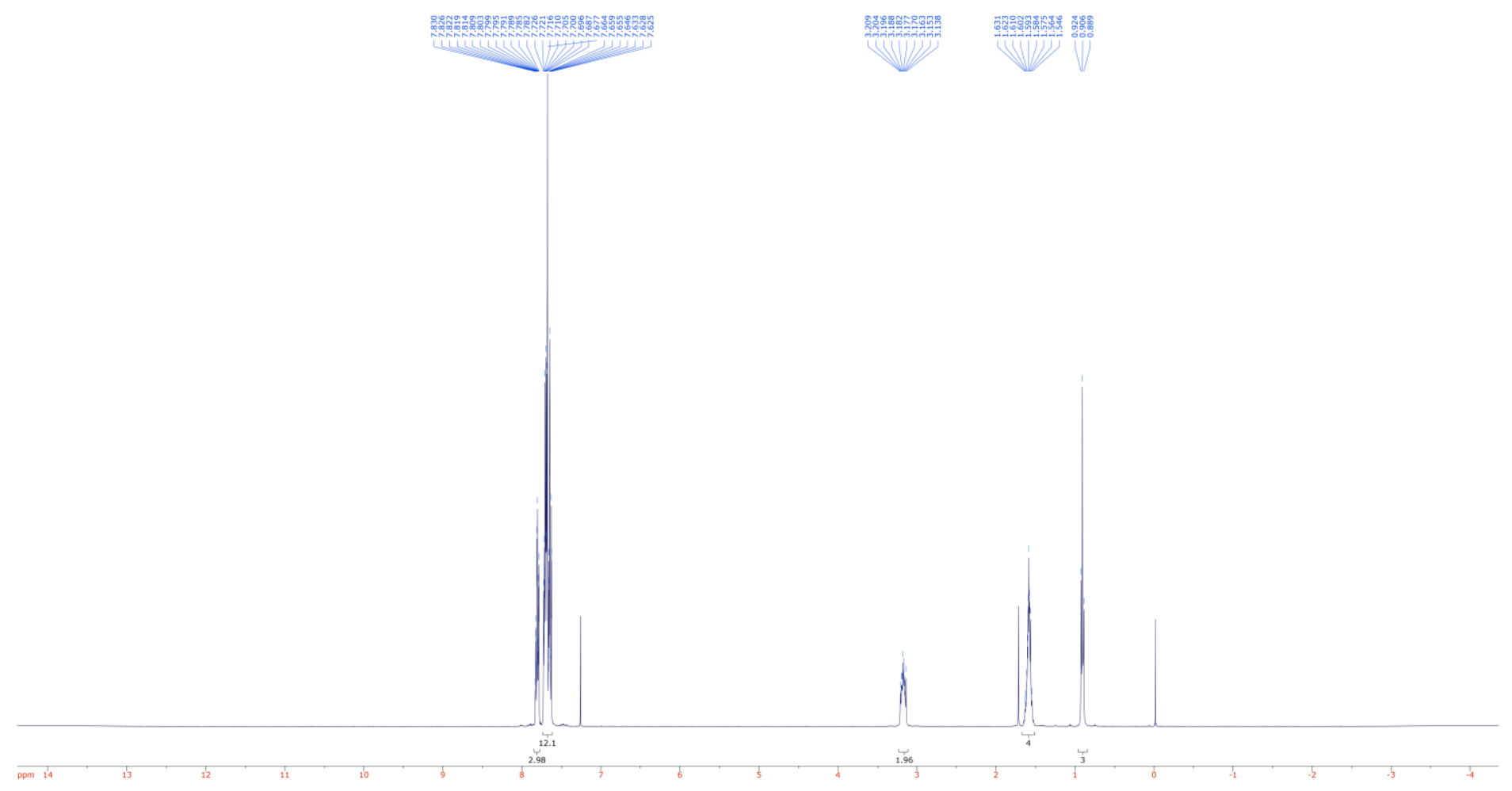

Figure S8: 13C NMR for Compound 3
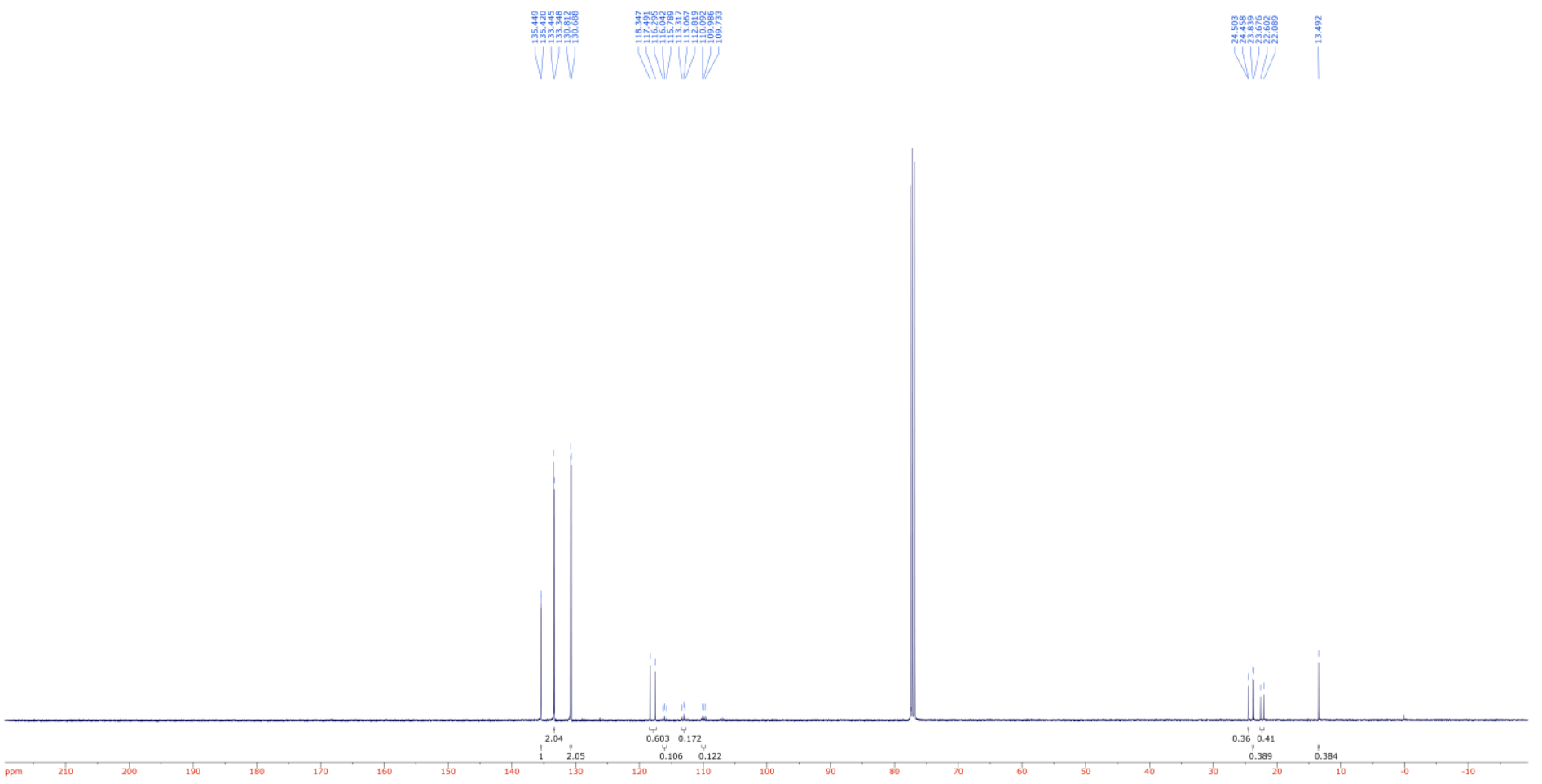
Figure S9: 19F NMR for Compound 3

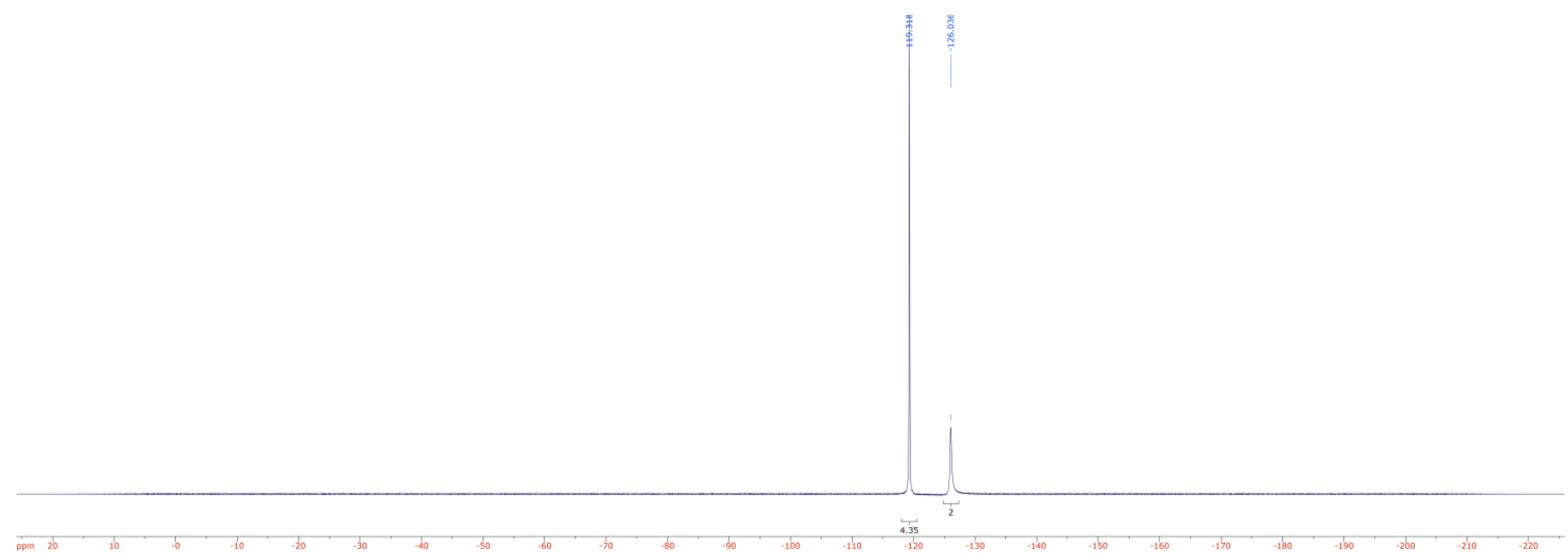

Figure S10: 1H NMR for Compound 4
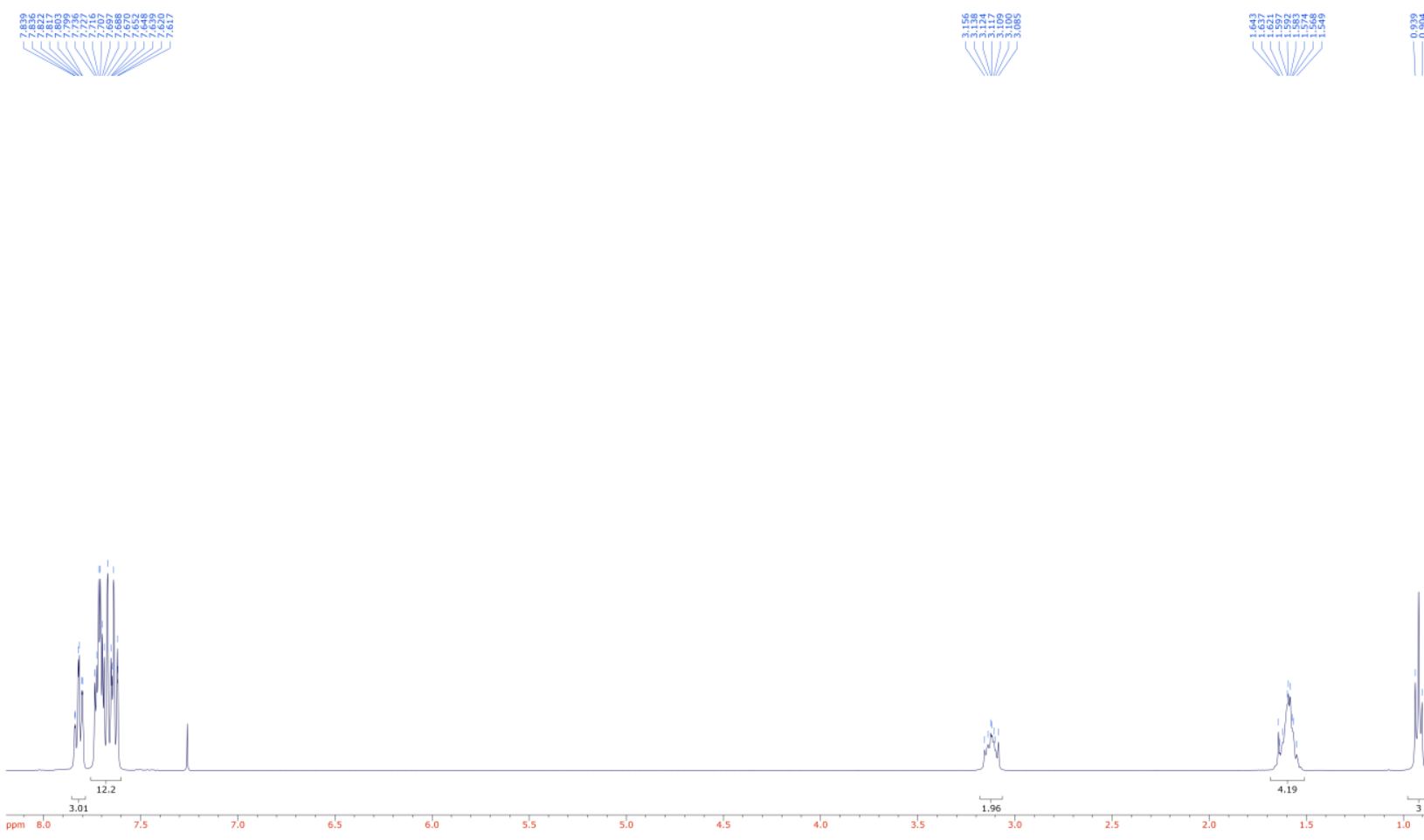
Figure S11 13C NMR for Compound 4

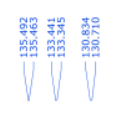

筑

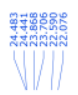

i

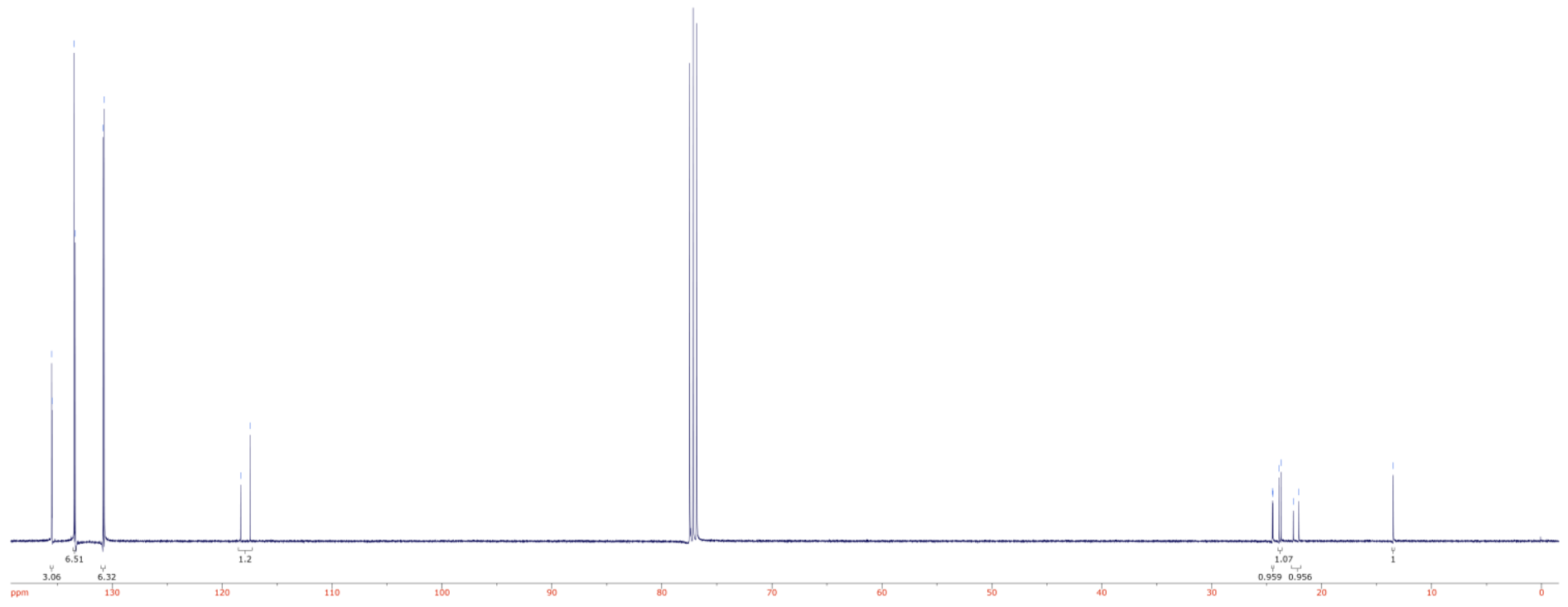

Figure S12 19F NMR for Compound 4

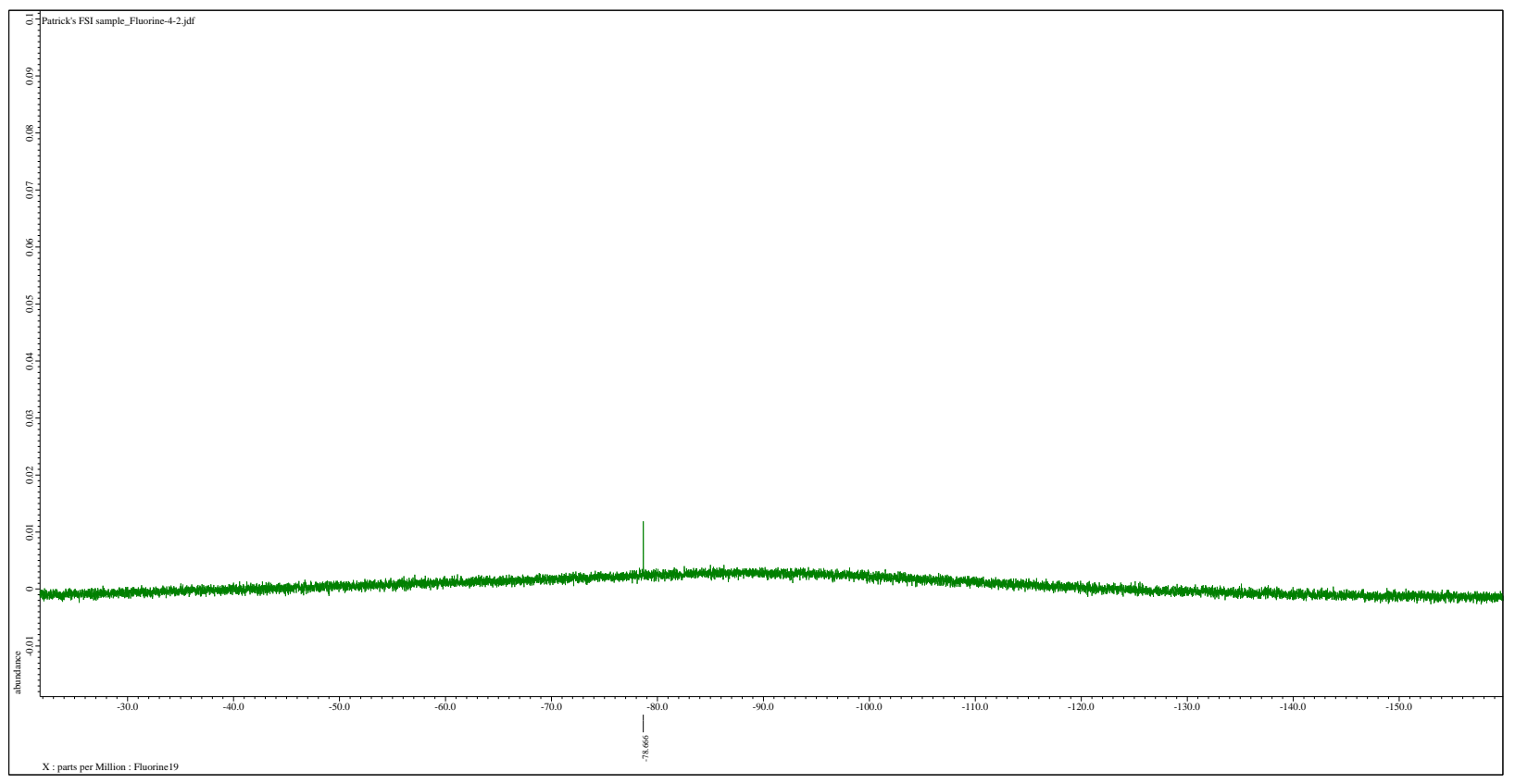

Sq 
Figure S13 DSC Traces for compound 1

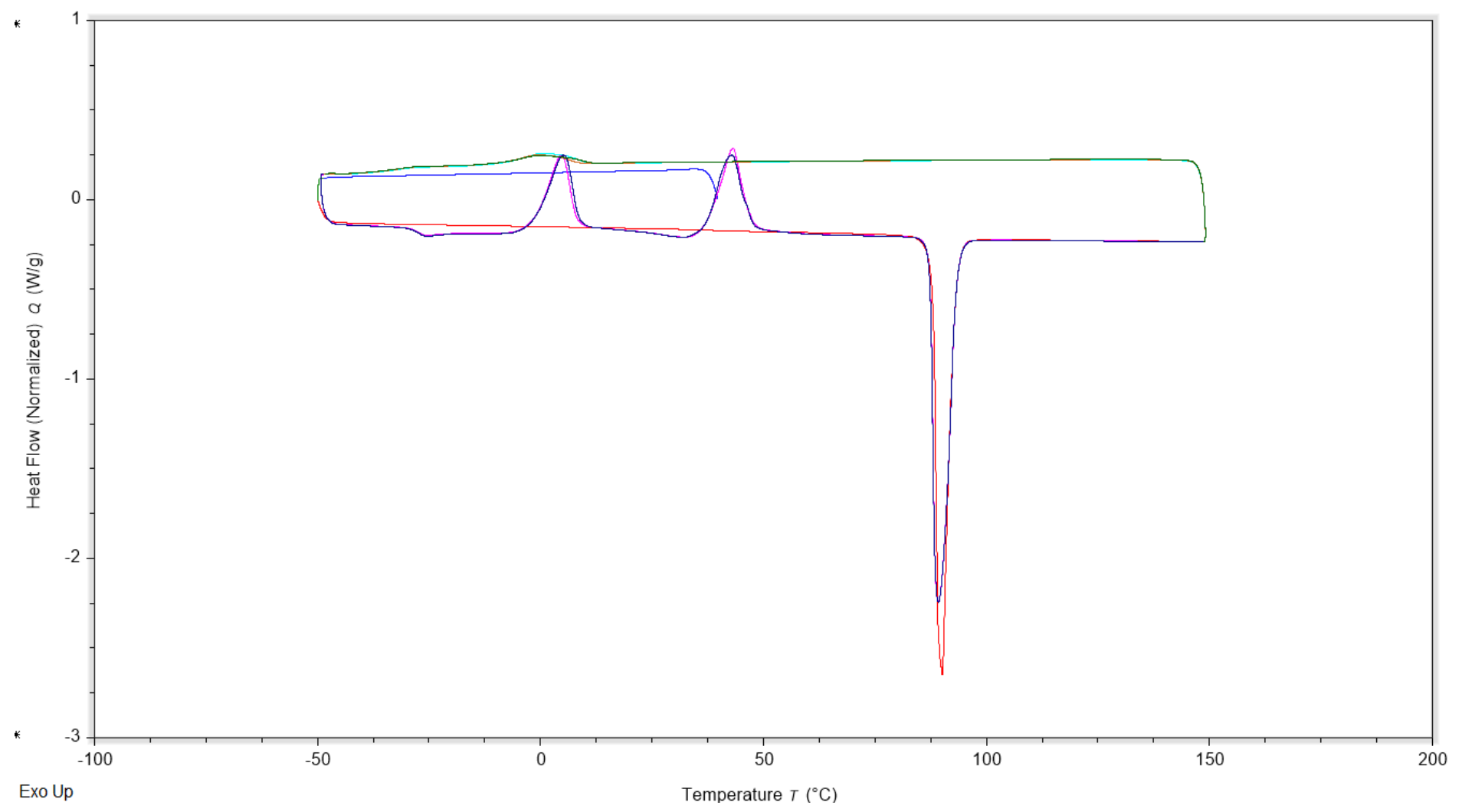

Figure S14 DSC Traces for compound 2

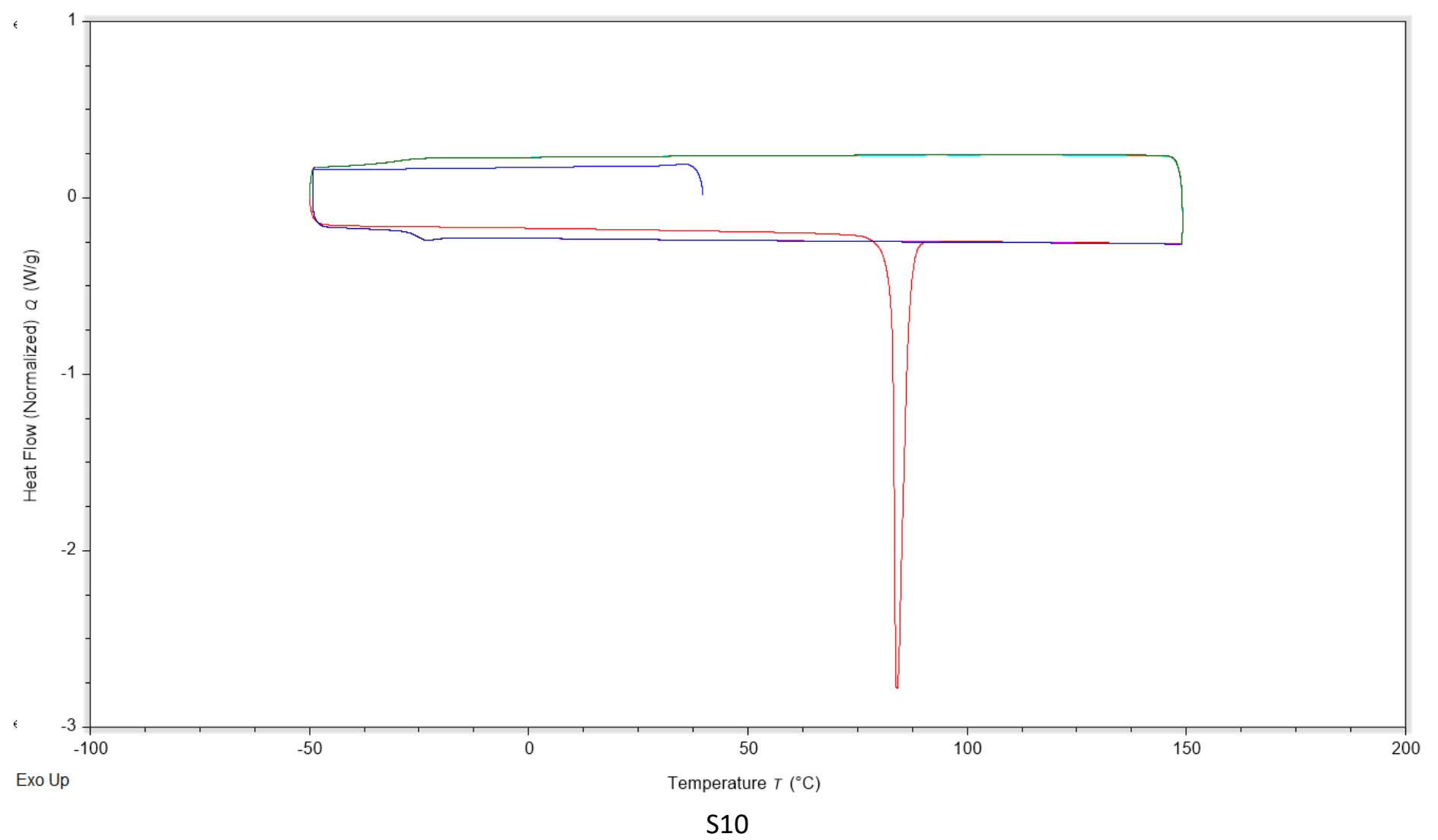


Figure S15 DSC Traces for compound 3

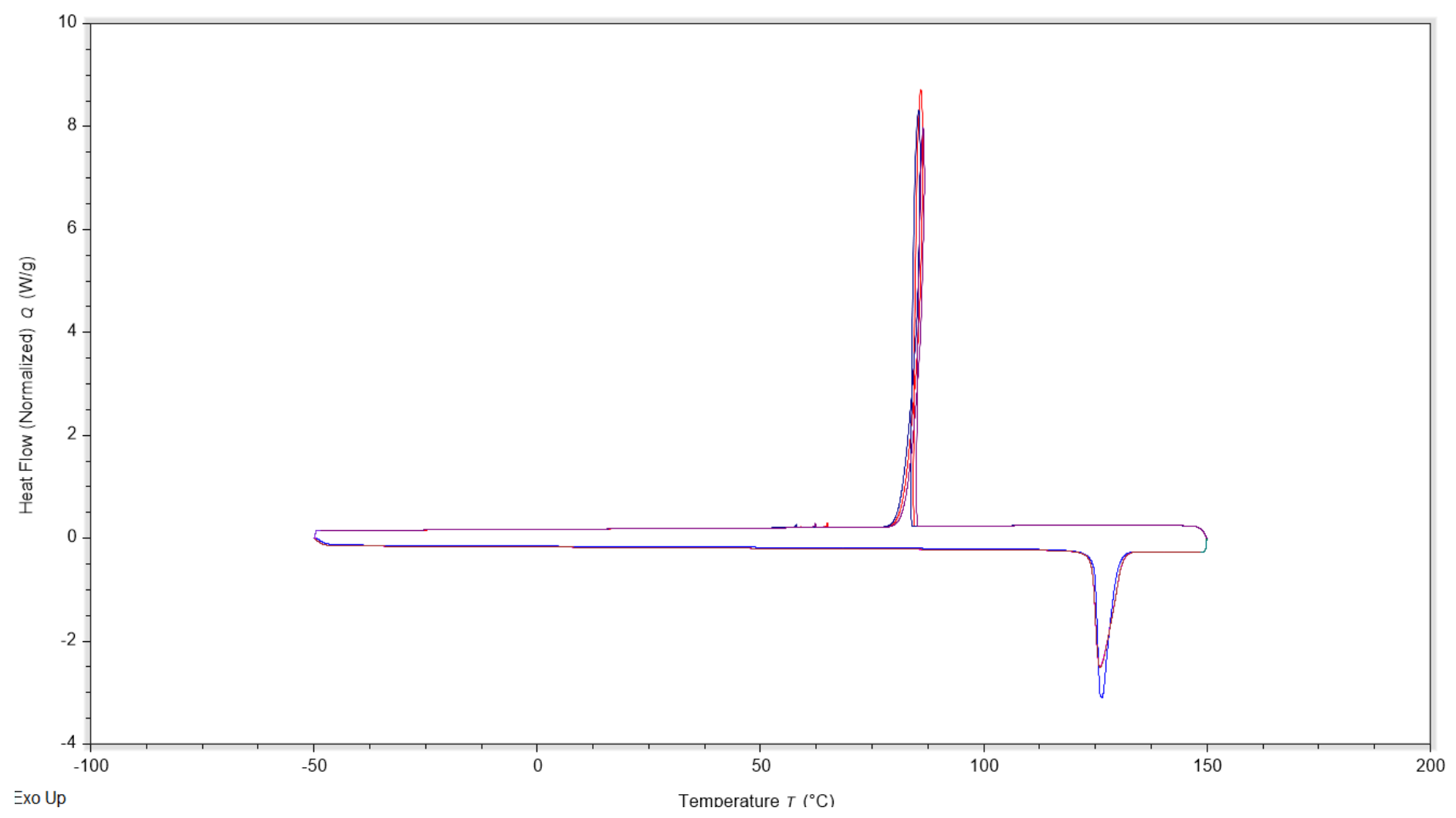

Figure S16 DSC Traces for compound 4

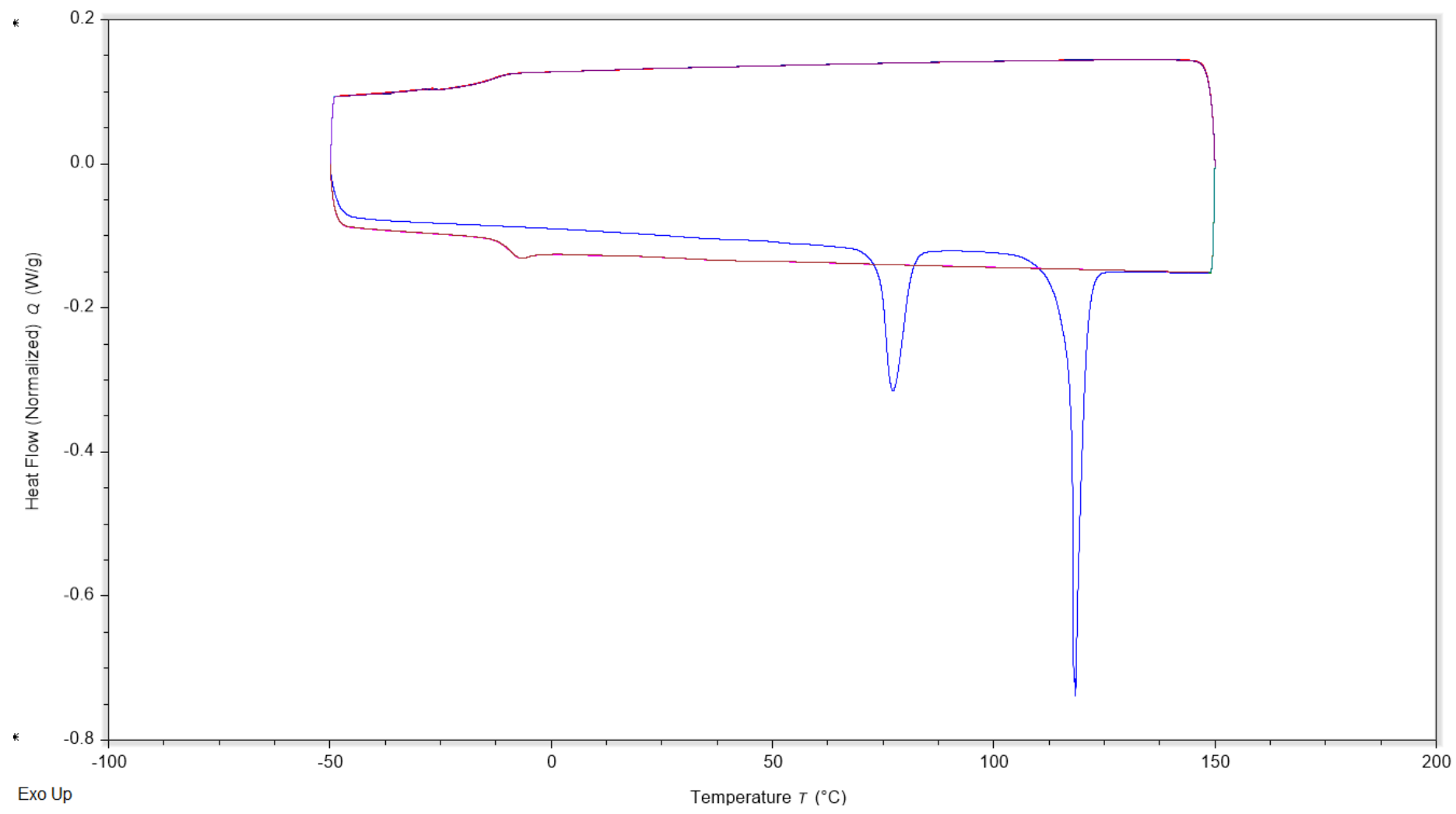

